\title{
Exact Standard Zeta Values of Siegel Modular Forms
}

\author{
Hidenori Katsurada
}

\section{CONTENTS}

1. Introduction

2. Pullback Formula for Siegel-Eisenstein Series

3. Exact Standard Zeta Values for $n=2$

4. Numerical Examples and Comments

Acknowledgments

References
2000 AMS Subject Classification: Primary 11F67

Keywords: Standard zeta values, Siegel modular forms
In this paper we give exact values of the standard zeta function for cuspidal Hecke eigenforms with respect to $\mathrm{Sp}_{2}(\mathbb{Z})$.

\section{INTRODUCTION}

For a cuspidal Hecke eigenform $f$ of weight $k$ with respect to $\operatorname{Sp}_{n}(\mathbb{Z})$, let $L(f, s, \underline{\mathrm{St}})$ be the standard zeta function of $f$. Then for some positive integer, the value

$$
\frac{L(f, m, \underline{\mathrm{St}})}{\langle f, f\rangle \pi^{-n(n+1) / 2+n k+(n+1) m}}
$$

is an algebraic number if all the Fourier coefficients of $f$ are algebraic, where $\langle f, f\rangle$ is the (unnormalized) Petersson product (cf. [Böcherer 85, Mizumoto 91]). In [Katsurada 08], we gave an explicit formula for computing this value in terms of Fourier coefficients of $f$ and some other elementary quantities. In this paper, we compute this value exactly in the case $n=2$. The main tool we use is the pullback formula for the Siegel-Eisenstein series due to [Garrett 84, Böcherer 85]. This method has been applied to the case of elliptic modular forms in [Katsurada 05]. To carry out the computation in the case of Siegel modular forms of degree 2, we need the explicit form of differential operators on the space of Siegel modular forms of degree 4 due to [Ibukiyama 99], and an explicit formula for global Siegel series for a half-integral matrix of degree 4 . The generating function of the differential operators has been given in [Ibukiyama 99], and by a direct but rather elaborate computation we can obtain an explicit form of them. An explicit formula for local Siegel series has been given in [Katsurada 99]. However, it seems rather difficult to use the formula directly for a practical computation. In this paper we present a trick that enables us to reduce the computation of global Siegel series of degree 4 to those of degree 2 .

The contents of this paper are as follows. In Section 2, we first review a result concerning Fourier coefficients of Siegel-Eisenstein series following [Katsurada 08], and explain the relation between the Siegel series and local 
densities. Next, we review a result concerning the pullback formula of Siegel-Eisenstein series due to Böcherer to obtain an exact value of the standard zeta function following [Katsurada 08]. In Section 3, we restrict ourselves to the case of Siegel modular forms of degree 2. First we present the trick for the computation of global Siegel series referred to above. Next we give an explicit formula for the differential operators acting on the space of Siegel modular forms of degree 4 due to [Ibukiyama 99], and give the main result of this paper, which enables us to obtain exact standard zeta values (cf. Theorem 3.8). In Section 4, we give numerical examples of such values and give some comments on the conjecture proposed in [Katsurada 08].

\subsection{Notation}

For a commutative ring $R$, we denote by $M_{m n}(R)$ the set of $(m, n)$-matrices with entries in $R$. In particular, put $M_{n}(R)=M_{n n}(R)$. For an $(m, n)$-matrix $X$ and an $(m, m)$-matrix $A$, we write $A[X]={ }^{t} X A X$, where ${ }^{t} X$ denotes the transpose of $X$. Let $a$ be an element of $R$. Then for an element $X$ of $M_{m n}(R)$ we often use the same symbol $X$ to denote the $\operatorname{coset} X \bmod a M_{m n}(R)$.

Put $\mathrm{GL}_{m}(R)=\left\{A \in M_{m}(R) \mid \operatorname{det} A \in R^{*}\right\}$, where $\operatorname{det} A$ denotes the determinant of a square matrix $A$, and $R^{*}$ denotes the unit group of $R$. Let $S_{n}(R)$ denote the set of symmetric matrices of degree $n$ with entries in $R$. Furthermore, for an integral domain $R$ of characteristic different from 2 , let $\mathcal{H}_{n}(R)$ denote the set of half-integral matrices of degree $n$ over $R$, that is, $\mathcal{H}_{n}(R)$ is the set of symmetric matrices of degree $n$ whose $(i, j)$-component belongs to $R$ or $\frac{1}{2} R$ according as $i=j$ or not.

For a subset $S$ of $M_{n}(R)$ we denote by $S^{\times}$the subset of $S$ consisting of nondegenerate matrices. In particular, if $S$ is a subset of $S_{n}(\mathbb{R})$ with $\mathbb{R}$ the field of real numbers, we denote by $S_{>0}$ (respectively $S_{\geq 0}$ ) the subset of $S$ consisting of positive definite (respectively positive semidefinite) matrices. Let $R^{\prime}$ be a subring of $R$. Two symmetric matrices $A$ and $A^{\prime}$ with entries in $R$ are said to be equivalent over $R^{\prime}$ and we write $A \widetilde{R^{\prime}} A^{\prime}$ if there is an element $X$ of $\mathrm{GL}_{n}\left(R^{\prime}\right)$ such that $A^{\prime}=A[X]$. We also write $A \sim A^{\prime}$ if there is no fear of confusion. For square matrices $X$ and $Y$ we write

$$
X \perp Y=\left(\begin{array}{cc}
X & O \\
O & Y
\end{array}\right)
$$

\section{PULLBACK FORMULA FOR SIEGEL-EISENSTEIN SERIES}

In this section we begin by reviewing the Fourier coefficients of Siegel-Eisenstein series following [Katsurada 08]. Furthermore, for later use, we consider the relation between Siegel series and local densities.

Put

$$
J_{n}=\left(\begin{array}{cc}
O_{n} & -1_{n} \\
1_{n} & O_{n}
\end{array}\right),
$$

where $1_{n}$ denotes the unit matrix of degree $n$. For a subring $K$ of $\mathbb{R}$ put

$$
\Gamma^{(n)}=\operatorname{Sp}_{n}(\mathbb{Z})=\left\{M \in \mathrm{GL}_{2 n}(\mathbb{Z}) \mid J_{n}[M]=J_{n}\right\} .
$$

Let $\mathbb{H}_{n}$ be Siegel's upper half-space. We denote by $\mathfrak{R}_{k}\left(\Gamma^{(n)}\right)$ (respectively $\mathfrak{R}_{k}^{\infty}\left(\Gamma^{(n)}\right)$ ) the space of holomorphic (respectively $C^{\infty}$ ) modular forms of weight $k$ with respect to $\Gamma^{(n)}$.

We denote by $\mathfrak{S}_{k}\left(\Gamma^{(n)}\right)$ the submodule of $\mathfrak{\Re}_{k}\left(\Gamma^{(n)}\right)$ consisting of cusp forms. For two $C^{\infty}$ modular forms $f$ and $g$ of weight $k$ with respect to $\Gamma^{(n)}$ we define the Petersson scalar product $\langle f, g\rangle$ as in [Katsurada 08].

For a positive integer $k \geq(n+1) / 2$ we define the Siegel-Eisenstein series $E_{n, k}(Z, s)$ of degree $n$ as

$$
\begin{aligned}
E_{n, k}(Z, s)= & \zeta(1-k-2 s) \prod_{i=1}^{[n / 2]} \zeta(1-2 k-4 s+2 i) \\
& \times \sum_{M \in \Gamma_{\infty}^{(n)} \backslash \Gamma^{(n)}} j(M, Z)^{-k}(\operatorname{det}(\Im(M(Z))))^{s},
\end{aligned}
$$

with $Z \in \mathbb{H}_{n}, s \in \mathbb{C}$, where $\zeta(*)$ is Riemann's zeta function, and

$$
\Gamma_{\infty}^{(n)}=\left\{\left(\begin{array}{cc}
* & * \\
O_{n} & *
\end{array}\right) \in \Gamma^{(n)}\right\} .
$$

Then $E_{n, k}(Z, s)$ is holomorphic at $s=0$ as a function of $s$, and $E_{n, k}(Z, 0)$ is holomorphic as a function of $Z$ unless $k=(n+2) / 2 \equiv 2 \bmod 4$ or $k=(n+3) / 2 \equiv 2 \bmod 4($ cf. [Shimura 83].)

From now on we assume that $E_{n, k}(Z, 0)$ is holomorphic as a function of $Z$ and write $E_{n, k}(Z)=E_{n, k}(Z, 0)$. To see the Fourier expansion of $E_{n, k}(Z, 0)$ for a prime number $p$ and a half-integral matrix $B$ of degree $n$ over $\mathbb{Z}_{p}$, define the local Siegel series $b_{p}(B, s)$ as in [Katsurada 08].

Let $m, n$ be nonnegative integers such that $m \geq n \geq 1$. For $A \in \mathcal{H}_{m}\left(\mathbb{Z}_{p}\right)$ and $B \in S_{n}\left(\mathbb{Q}_{p}\right)$ we define the local density $\alpha_{p}(A, B)$ and the primitive local density $\beta_{p}(A, B)$ by

$$
\alpha_{p}(A, B)=2^{\delta_{m n}} \lim _{e \rightarrow \infty} p^{(-m n+n(n+1) / 2) e} \# \mathcal{A}_{e}(A, B)
$$


and

$$
\beta_{p}(A, B)=2^{\delta_{m n}} \lim _{e \rightarrow \infty} p^{(-m n+n(n+1) / 2) e} \# \mathcal{B}_{e}(A, B),
$$

where $\delta_{m n}$ is Kronecker's delta,

$$
\begin{aligned}
\mathcal{A}_{e}(A, B) & =\left\{X \in M_{m n}\left(\mathbb{Z}_{p}\right) / p^{e} M_{m n}\left(\mathbb{Z}_{p}\right) \mid A[X]-B\right. \\
& \left.\in p^{e} \mathcal{H}_{n}\left(\mathbb{Z}_{p}\right)\right\}
\end{aligned}
$$

and

$$
\mathcal{B}_{e}(A, B)=\left\{X \in \mathcal{A}_{e}(A, B) \mid \operatorname{rank}_{\mathbb{Z}_{p} / p \mathbb{Z}_{p}}(X)=n\right\} .
$$

We define $\chi_{p}(a)$ for $a \in \mathbb{Q}_{p} \backslash\{0\}$ as follows;

$$
\chi_{p}(a)= \begin{cases}+1 & \text { if } \mathbb{Q}_{p}(\sqrt{a})=\mathbb{Q}_{p} \\ -1 & \text { if } \mathbb{Q}_{p}(\sqrt{a}) / \mathbb{Q}_{p} \text { is quadratic unramified } \\ 0 & \text { if } \mathbb{Q}_{p}(\sqrt{a}) / \mathbb{Q}_{p} \text { is quadratic ramified. }\end{cases}
$$

For a half-integral matrix $B$ of even degree $n$ we define $\xi_{p}(B)$ by

$$
\xi_{p}(B)=\chi_{p}\left((-1)^{n / 2} \operatorname{det} B\right) .
$$

Let $B \in \mathcal{H}_{n}(\mathbb{Z})_{>0}$ with $n$ even. Then we can

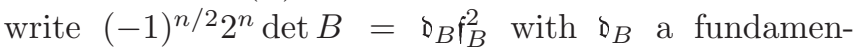
tal discriminant and $\mathrm{f}_{B} \in \mathbb{Z}_{>0}$. Furthermore, let $\chi_{B}=\left(\frac{\searrow_{B}}{*}\right)$ be the Kronecker character corresponding to $\mathbb{Q}\left(\sqrt{(-1)^{n / 2} \operatorname{det} B}\right) / \mathbb{Q}$. We note that we have $\chi_{B}(p)=$ $\xi_{p}(B)$ for any prime $p$. Let

$$
H_{k}=\overbrace{H \perp \cdots \perp H}^{k} \quad \text { with } H=\left(\begin{array}{cc}
0 & 1 / 2 \\
1 / 2 & 0
\end{array}\right) .
$$

For a nondegenerate half-integral matrix $B$ of degree $n$ over $\mathbb{Z}_{p}$ we define a polynomial $\gamma_{p}(B, X)$ in $X$ by

$$
\begin{aligned}
\gamma_{p}(B, X) \\
=\left\{\begin{array}{c}
(1-X) \prod_{i=1}^{n / 2}\left(1-p^{2 i} X^{2}\right)\left(1-p^{n / 2} \xi_{p}(B) X\right)^{-1} \\
\quad \text { if } n \text { is even, } \\
(1-X) \prod_{i=1}^{(n-1) / 2}\left(1-p^{2 i} X^{2}\right) \\
\text { if } n \text { is odd. }
\end{array}\right.
\end{aligned}
$$

Then the following lemma is well known (e.g., [Kitaoka 84, Lemma 1]).

Lemma 2.1. For a nondegenerate half-integral matrix $B$ of degree $n$ over $\mathbb{Z}_{p}$ there exists a unique polynomial $F_{p}(B, X)$ in $X$ over $\mathbb{Z}$ with constant term 1 such that

$$
b_{p}(B, s)=\gamma_{p}\left(B, p^{-s}\right) F_{p}\left(B, p^{-s}\right) .
$$

Furthermore, for any positive integer $k \geq n / 2$ and a halfintegral matrix $A$ of degree $2 k$ over $\mathbb{Z}_{p}$ such that $2 A$ is unimodular, we have

$$
\alpha_{p}(A, B)=F_{p}\left(B, \xi_{p}(A) p^{-k}\right) \gamma_{p}\left(B, \xi_{p}(A) p^{-k}\right)
$$

and in particular,

$$
\alpha_{p}\left(H_{k}, B\right)=F_{p}\left(B, p^{-k}\right) \gamma_{p}\left(B, p^{-k}\right)
$$

Remark 2.2. For an element $B \in \mathcal{H}_{n}\left(\mathbb{Z}_{p}\right)$ of rank $m \geq 0$, there exists an element $\tilde{B} \in \mathcal{H}_{m}\left(\mathbb{Z}_{p}\right) \cap \mathrm{GL}_{m}\left(\mathbb{Q}_{p}\right)$ such that $B \sim \tilde{B} \perp O_{n-m}$. We note that $b_{p}(\tilde{B}, s)$ does not depend on the choice of $\tilde{B}$ (cf. [Kitaoka 84]). Thus we write this as $b_{p}^{(m)}(B, s)$. Furthermore, $F_{p}(\tilde{B}, X)$ does not depend on the choice of $\tilde{B}$. Then we put $F_{p}^{(m)}(B, X)=$ $F_{p}(\tilde{B}, X)$. For an element $B \in \mathcal{H}_{n}(\mathbb{Z})_{\geq 0}$ of rank $m \geq 0$, there exists an element $\tilde{B} \in \mathcal{H}_{m}(\mathbb{Z})_{>0}$ such that $B \sim$ $\tilde{B} \perp O_{n-m}$. Then $\operatorname{det} \tilde{B}$ does not depend on the choice of $B$. Therefore, we put $\operatorname{det}^{(m)} B=\operatorname{det} \tilde{B}$. Similarly, we write $\chi_{B}^{(m)}=\chi_{\tilde{B}}$ if $m$ is even.

Now for a positive semidefinite half-integral matrix $B$ of degree $2 n$ and of rank $m$, we put

$$
\begin{aligned}
& c_{2 n, l}(B)=2^{[(m+1) / 2]} \prod_{p} F_{p}^{(m)}\left(B, p^{l-m-1}\right) \\
& \quad \times\left\{\begin{array}{l}
\prod_{i=m / 2+1}^{n} \zeta(1+2 i-2 l) L\left(1+m / 2-l, \chi_{B}^{(m)}\right) \\
\text { if } m \text { is even, } \\
\prod_{i=(m+1) / 2}^{n} \zeta(1+2 i-2 l) \\
\text { if } m \text { is odd. }
\end{array}\right.
\end{aligned}
$$

Here we make the convention $F_{p}^{(m)}\left(B, p^{l-m-1}\right)=1$ and $L\left(1+m / 2-l, \chi_{B}^{(m)}\right)=\zeta(1-l)$ if $m=0$. Then we have the following result.

Theorem 2.3. Let $l$ be a positive even integer. Assume that $l \geq n+3$ or $l \geq n+1$ according as $n \equiv 1 \bmod 4$ or not. Then we have

$$
E_{2 n, l}(Z)=\sum_{B \in \mathcal{H}_{2 n}(\mathbb{Z})_{\geq 0}} c_{2 n, l}(B) \mathbf{e}(\operatorname{tr}(B Z)),
$$

where $\mathbf{e}(x)=\exp (2 \pi \sqrt{-1} x)$ for a complex number $x$, and tr denotes the trace of a matrix.

Now we review the pullback formula of SiegelEisenstein series following [Katsurada 08, Sections 3, 4]. Let $\mathbf{L}_{n}^{\prime}=\mathbf{L}_{\mathbb{Q}}\left(\operatorname{GSp}_{n}(\mathbb{Q})^{+} \cap M_{2 n}(\mathbb{Z}), \Gamma^{(n)}\right)$ denote the Hecke ring over $\mathbb{Z}$ associated with the Hecke pair $\left(\operatorname{GSp}_{n}(\mathbb{Q})^{+} \cap M_{2 n}(\mathbb{Z}), \Gamma^{(n)}\right)$. Furthermore, for a prime number $p$ put

$$
\begin{aligned}
& \operatorname{GSp}_{n}\left(\mathbb{Q}_{p}\right) \\
& \quad=\left\{M \in \mathrm{GL}_{2 n}\left(\mathbb{Q}_{p}\right) ; J_{n}[M]=\kappa(M) J_{n}, \kappa(M) \in \mathbb{Q}_{p}^{\times}\right\},
\end{aligned}
$$


and let $\mathbf{L}_{n p}=\mathbf{L}\left(\mathrm{GSp}_{n}\left(\mathbb{Q}_{p}\right), \mathrm{GSp}_{n}\left(\mathbb{Q}_{p}\right) \cap \mathrm{GL}_{2 n}\left(\mathbb{Z}_{p}\right)\right)$ be the Hecke algebra associated with the pair $\left(\mathrm{GSp}_{n}\left(\mathbb{Q}_{p}\right), \mathrm{GSp}_{n}\left(\mathbb{Q}_{p}\right) \cap \mathrm{GL}_{2 n}\left(\mathbb{Z}_{p}\right)\right)$.

Now assume that $f$ is a Hecke eigenform, namely, a common eigenfunction of all Hecke operators. For each prime number $p$, let $\alpha_{0}(p), \alpha_{1}(p), \ldots, \alpha_{n}(p)$ be the Satake parameters of $\mathbf{L}_{n p}$ determined by $f$. We then define the standard zeta function $L(f, s, \underline{\mathrm{St}})$ by

$$
\begin{aligned}
& L(f, s, \underline{\mathrm{St}}) \\
& \quad=\prod_{p} \prod_{i=1}^{n}\left\{\left(1-p^{-s}\right)\left(1-\alpha_{i}(p) p^{-s}\right)\left(1-\alpha_{i}(p)^{-1} p^{-s}\right)\right\}^{-1} .
\end{aligned}
$$

Now let $\stackrel{\circ}{\mathcal{D}}_{n, l}^{\nu}$ be the differential operator from $\mathfrak{R}_{l}^{\infty}\left(\Gamma^{(2 n)}\right)$ to $\mathfrak{M}_{l+\nu}^{\infty}\left(\Gamma^{(n)}\right) \otimes \mathfrak{M}_{l+\nu}^{\infty}\left(\Gamma^{(n)}\right)$ in [Böcherer and Schmidt 00].

Let $E_{2 n, l}(Z)=E_{2 n, l}(Z, 0)$ be the Eisenstein series as above. We then define $\mathcal{E}_{2 n, l, k}\left(z_{1}, z_{2}\right)$ as

$$
\begin{aligned}
\mathcal{E}_{2 n, l, k}\left(z_{1}, z_{2}\right)= & (-1)^{l / 2+1} 2^{-n}(2 \pi \sqrt{-1})^{(l-k) n}(l-n) \\
& \times \stackrel{\circ}{\mathcal{D}}_{n, l}^{k-l}\left(E_{2 n, l}\right)\left(z_{1}, z_{2}\right),
\end{aligned}
$$

where $z_{1}, z_{2} \in \mathbb{H}_{n}$. Let

$$
f(z)=\sum_{A \in \mathcal{H}_{n}(\mathbb{Z})_{>0}} a(A) \mathbf{e}(\operatorname{tr}(A z))
$$

be a Hecke eigenform in $\mathfrak{\subseteq}_{k}\left(\Gamma^{(n)}\right)$. For a positive integer $m \leq k-n$ such that $m \equiv n \bmod 2$ put

$$
\begin{aligned}
\Lambda(f, m, \underline{\mathrm{St}})= & (-1)^{n(-m+1) / 2+1} 2^{-4 k n+3 n^{2}+n+(n-1) m+2} \\
& \times \Gamma(m+1) \prod_{i=1}^{n} \Gamma(2 k-n-i) \\
& \times \frac{L(f, m, \underline{\mathrm{St}})}{\langle f, f\rangle \pi^{-n(n+1) / 2+n k+(n+1) m}} .
\end{aligned}
$$

For two positive semidefinite half-integral matrices $A_{1}, A_{2}$ of degree $n$, put

$$
\begin{aligned}
\epsilon_{l, k}\left(A_{1}, A_{2}\right)= & \sum_{A_{2}-\frac{1}{4} A_{1}^{-1}[R] \geq 0} \tilde{c}_{2 n, l}\left(\left(\begin{array}{cc}
A_{1} & R / 2 \\
{ }^{t} R / 2 & A_{2}
\end{array}\right)\right) \\
& \times Q_{n, l}^{k-l}\left(\left(\begin{array}{cc}
A_{1} & R / 2 \\
{ }^{t} R / 2 & A_{2}
\end{array}\right)\right)
\end{aligned}
$$

where $\tilde{c}_{2 n, l}(A)=(-1)^{l / 2+1} 2^{-n}(l-n) c_{2 n, l}(A)$ for $A \in$ $\mathcal{H}_{2 n}(\mathbb{Z})_{\geq 0}$, and $Q_{n, l}^{k-l}$ is the polynomial defined in [Katsurada 08, Section 3]. Furthermore, for each positive semidefinite half-integral matrix $A_{1}$, put

$$
\mathcal{F}_{l, k ; A_{1}}\left(z_{2}\right)=\sum_{A_{2} \in \mathcal{H}_{n}(\mathbb{Z})_{\geq 0}} \epsilon_{l, k}\left(A_{1}, A_{2}\right) \mathbf{e}\left(\operatorname{tr}\left(A_{2} z_{2}\right)\right) .
$$

We note that we have

$$
\begin{aligned}
\mathcal{E}_{2 n, l, k}\left(z_{1}, z_{2}\right) & \sum_{A_{1} \in \mathcal{H}_{n}(\mathbb{Z})_{\geq 0}} \sum_{A_{2} \in \mathcal{H}_{n}(\mathbb{Z})_{\geq 0}} \epsilon_{l, k}\left(A_{1}, A_{2}\right) \mathbf{e}\left(\operatorname{tr}\left(A_{1} z_{1}+A_{2} z_{2}\right)\right) .
\end{aligned}
$$

Thus $\mathcal{F}_{l, k ; A_{1}}\left(z_{2}\right)$ belongs to $\mathfrak{R}_{k}\left(\Gamma^{(n)}\right)$, and

$$
\mathcal{E}_{2 n, l, k}\left(z_{1}, z_{2}\right)=\sum_{A_{1} \in \mathcal{H}_{n}(\mathbb{Z})_{\geq 0}} \mathcal{F}_{l, k ; A_{1}}\left(z_{2}\right) \mathbf{e}\left(\operatorname{tr}\left(A_{1} z_{1}\right)\right)
$$

(cf. [Katsurada 08, Section 3]). In particular, if $l<k$, then $\mathcal{F}_{l, k ; A_{1}}\left(z_{2}\right)$ belongs to $\mathfrak{S}_{k}\left(\Gamma^{(n)}\right)$, and

$$
\mathcal{E}_{2 n, l, k}\left(z_{1}, z_{2}\right)=\sum_{A_{1} \in \mathcal{H}_{n}(\mathbb{Z})>0} \mathcal{F}_{l, k ; A_{1}}\left(z_{2}\right) \mathbf{e}\left(\operatorname{tr}\left(A_{1} z_{1}\right)\right) .
$$

Take an orthogonal basis $\left\{f_{i}\right\}_{i=1}^{d_{1}}$ of $\mathfrak{\Xi}_{k}\left(\Gamma^{(n)}\right)$ consisting of Hecke eigenforms. Write

$$
f_{i}(z)=\sum_{A \in \mathcal{H}_{n}(\mathbb{Z})_{>0}} a_{i}(A) \mathbf{e}(\operatorname{tr}(A z)) .
$$

Now we have the following proposition.

Proposition 2.4. [Katsurada 08, Theorem 4.4] Let $l, k$, and $n$ be a positive integers. Assume that $k$ and $l+n$ are even, and $3 \leq l \leq k-n-2$ or $1 \leq l \leq k-n-2$ according as $n \equiv 1 \bmod 4$ or not. Then for any positive definite half-integral matrix $A_{1}$ of degree $n$, we have

$$
\mathcal{F}_{l+n, k ; A_{1}}(z)=\sum_{i=1}^{d_{1}} \Lambda\left(f_{i}, l, \underline{\text { St }}\right) a_{i}\left(A_{1}\right) \overline{f_{i}(-\bar{z})} .
$$

Remark 2.5. There are some typographical errors in [Katsurada 08, p. 105]:

- line 9: For $(2 \pi \sqrt{-1})^{l-k}, \operatorname{read}(2 \pi \sqrt{-1})^{(l-k) n}$;

- line 12: For $(-1)^{n(m+1) / 2+1}, \operatorname{read}(-1)^{n(-m+1) / 2+1}$;

- line 13: For $\Gamma(m), \operatorname{read} \Gamma(m+1)$.

For a prime number $p$, let $T(p)$ be the element of $\mathbf{L}_{n}^{\prime}$ defined by $T(p)=\Gamma^{(n)}\left(1_{n} \perp p 1_{n}\right) \Gamma^{(n)}$. For each nonnegative integer $i$ and $A_{1} \in \mathcal{H}_{n}(\mathbb{Z})_{>0}$, write $\mathcal{F}_{l+n, k ; A_{1}} \mid T(p)^{i}(z)$ as

$\mathcal{F}_{l+n, k ; A_{1}} \mid T(p)^{i}(z)=\sum_{A \in \mathcal{H}_{n}(\mathbb{Z})_{>0}} \epsilon_{l+n, k}\left(i, A_{1}, A\right) \mathbf{e}(\operatorname{tr}(A z))$. 
Furthermore, write

$$
f_{j} \mid T(p)(z)=\lambda_{j} f_{j}(z)
$$

Thus by Proposition 2.4 we have the following result.

Lemma 2.6. Under the above notation and assumption, we have

$$
\epsilon_{l+n, k}\left(i, A_{1}, A\right)=\sum_{j=1}^{d} \lambda_{j}^{i} \Lambda\left(f_{j}, l, \underline{\mathrm{St}}\right) a_{j}\left(A_{1}\right) \overline{a_{j}(A)}
$$

for any nonzero integer $i$ and $A \in \mathcal{H}_{n}(\mathbb{Z})_{>0}$.

From now on, we assume that $\mathfrak{S}_{k}\left(\Gamma^{(n)}\right)$ has the multiplicity-one condition. Namely, we assume that a Hecke eigenform in $\mathfrak{S}_{k}\left(\Gamma^{(n)}\right)$ is uniquely determined, up to a constant multiple, by its eigenvalues of Hecke operators. First we normalize the standard zeta value $\Lambda(f, l, \underline{\mathrm{St}})$ for a Hecke eigenform $f$ in $\mathfrak{S}_{k}\left(\Gamma^{(n)}\right)$ following [Katsurada 08]. We define the following quantities: For a Hecke eigenform $f(z)=\sum_{A} a_{f}(A) \mathbf{e}(\operatorname{tr}(A z))$ in $\mathfrak{S}_{k}\left(\Gamma^{(n)}\right)$, let $\mathfrak{I}_{f}$ be the $\mathfrak{D}_{\mathbb{Q}(f)}$-module generated by all $a_{f}(A)$ 's. Then, multiplying by a suitable constant $c$, we may assume that all $a_{f}(A)$ 's are elements of $\mathbb{Q}(f)$ with bounded denominator. Then $\mathfrak{I}_{f}$ is a fractional ideal in $\mathbb{Q}(f)$, and therefore so is $\Lambda(f, l, \underline{\mathrm{St}}) \mathfrak{I}_{f}^{2}$ if $l$ satisfies the conditions in Proposition 2.4. We note that this fractional ideal does not depend on the choice of $c$. In particular, these values are uniquely determined by the system of eigenvalues of $f$. We also note that the value $N_{\mathbb{Q}(f)}(\Lambda(f, l, \underline{\mathrm{St}})) N\left(\mathfrak{I}_{f}\right)^{2}$ does not depend on the choice of $c$, where $N\left(\mathfrak{I}_{f}\right)$ is the norm of the ideal $\mathfrak{I}_{f}$.

Theorem 2.7. In addition to the above assumption, let $f=f_{1}, \lambda=\lambda_{1}, a\left(A_{1}\right)=a_{1}\left(A_{1}\right), a(A)=a_{1}(A)$, $K=\mathbb{Q}(f)$, and $e_{i}=\epsilon_{l+n, k}\left(i, A_{1}, A\right)$. Furthermore, let $\Phi(X)=\Phi_{T(p)}(X)=\sum_{i=0}^{d} b_{d-i} X^{i}$ be the characteristic polynomial of $T(p)$ in $\mathfrak{S}_{k}\left(\Gamma^{(n)}\right)$. Put $\Lambda^{*}(f, l, \mathrm{St})=$ $N_{\mathbb{Q}(f)}(\Lambda(f, l, \underline{\mathrm{St}})) N\left(\mathfrak{I}_{f}\right)^{2}$ and assume that $\Phi^{\prime}(\lambda) \neq 0$, and $a\left(A_{1}\right) a(A) \neq 0$.

Then for any positive integer $l$ satisfying the conditions in Proposition 2.4, we have

$$
\begin{aligned}
\Lambda^{*}(f, l, \underline{\mathrm{St}})= & N_{K / \mathbb{Q}}\left(\frac{\sum_{i=0}^{d-1} \sum_{j=i}^{d-1} e_{d-1-j} b_{j-i} \lambda^{i}}{\Phi^{\prime}(\lambda)}\right) \\
& \times \frac{N\left(\mathfrak{I}_{f}\right)^{2}}{N_{K / \mathbb{Q}}\left(a\left(A_{1}\right) a(A)\right)} .
\end{aligned}
$$

Proof: By Lemma 2.6, we have

$$
e_{i}=\sum_{j=1}^{d} \lambda_{j}^{i} \Lambda\left(f_{j}, l, \underline{\mathrm{St}}\right) a_{j}\left(A_{1}\right) \overline{a_{j}(A)}
$$

for each $i=0, \ldots, d-1$. Then by [Goto 98, Lemma 2.2], we have

$$
\Lambda(f, l, \underline{\mathrm{St}}) a\left(A_{1}\right) a(A)=\frac{\sum_{i=0}^{d-1} \sum_{j=i}^{d-1} e_{d-1-j} b_{j-i} \lambda^{i}}{\Phi^{\prime}(\lambda)} .
$$

The assertion follows immediately.

Using the above theorem, we can in principle obtain standard zeta values of a Hecke eigenform in $\mathfrak{\Im}_{k}\left(\Gamma^{(n)}\right)$. However, to make the computation explicit, we need to compute the Fourier coefficients of the Siegel-Eisenstein series of degree $2 n$ and the differential operators explicitly. We will do this in the case $n=2$ in the next sections.

\section{EXACT STANDARD ZETA VALUES FOR $n=2$}

In this section we obtain a useful formula for computing exact standard zeta values in the case of degree 2 . The following lemma can easily be proved (e.g., [Katsurada 99, Proposition 2.2]).

Lemma 3.1. Let $n=n_{1}+n_{2}$ with $n_{1}$ even. Let $A_{11} \in$ $\mathcal{H}_{n_{1}}\left(\mathbb{Z}_{p}\right) \cap \frac{1}{2} \mathrm{GL}_{n_{1}}\left(\mathbb{Z}_{p}\right)$ and $A_{22} \in \mathcal{H}_{n_{2}}\left(\mathbb{Z}_{p}\right) \cap \mathrm{GL}_{n_{2}}\left(\mathbb{Q}_{p}\right)$. Then for any $l \geq n$ we have

$\alpha_{p}\left(H_{l}, A_{11} \perp A_{22}\right)=\beta_{p}\left(H_{l}, A_{11}\right) \alpha_{p}\left(H_{l-n_{1}} \perp\left(-A_{11}\right), A_{22}\right)$.

Proposition 3.2. Let $n_{1}$ be an even integer. Let $A_{11} \in$ $\mathcal{H}_{n_{1}}\left(\mathbb{Z}_{p}\right) \cap \frac{1}{2} \mathrm{GL}_{n_{1}}\left(\mathbb{Z}_{p}\right)$ and $A_{22} \in \mathcal{H}_{n_{2}}\left(\mathbb{Z}_{p}\right)$. Let $m$ be the rank of $A_{22}$. Then we have

$$
F_{p}^{\left(n_{1}+m\right)}\left(A_{11} \perp A_{22}, X\right)=F_{p}^{(m)}\left(A_{22}, \xi_{p}\left(A_{11}\right) p^{n_{1} / 2} X\right) .
$$

Proof: We may assume that $A_{22}$ is nondegenerate. By Lemma 2.1 , for any $l \geq n_{1}+n_{2}$ we have

$\alpha_{p}\left(H_{l}, A_{11} \perp A_{22}\right)=\gamma_{p}\left(A_{11} \perp A_{22}, p^{-l}\right) F_{p}\left(A_{11} \perp A_{22}, p^{-l}\right)$.

By Lemma 3.1, we have

$\alpha_{p}\left(H_{l}, A_{11} \perp A_{22}\right)=\beta_{p}\left(H_{l}, A_{11}\right) \alpha_{p}\left(H_{l-n_{1}} \perp\left(-A_{11}\right), A_{22}\right)$.

Again by Lemma 2.1, we have

$$
\begin{aligned}
\alpha_{p}\left(H_{l-n_{1}} \perp\left(-A_{11}\right), A_{22}\right)= & \gamma_{p}\left(A_{22}, \xi_{p}\left(A_{11}\right) p^{n_{1} / 2-l}\right) \\
& \times F_{p}\left(A_{22}, \xi_{p}\left(A_{11}\right) p^{n_{1} / 2-l}\right) .
\end{aligned}
$$


Furthermore, we have

$$
\begin{aligned}
& \beta_{p}\left(H_{l}, A_{11}\right) \\
& \quad=\left(1-p^{-l}\right) \prod_{i=1}^{n_{1} / 2}\left(1-p^{2 i-2 l}\right)\left(1-p^{n_{1} / 2-l} \xi_{p}\left(A_{11}\right)\right)^{-1}
\end{aligned}
$$

(e.g., [Kitaoka 93]), and by definition we have

$$
\begin{aligned}
& \gamma_{p}\left(A_{11} \perp A_{22}, p^{-l}\right) \\
& \quad=\beta_{p}\left(H_{l}, A_{11}\right) \gamma_{p}\left(A_{22}, \xi_{p}\left(A_{11}\right) p^{n_{1} / 2-l}\right) .
\end{aligned}
$$

Thus the assertion holds.

\section{Corollary 3.3. Let}

$A=\left(\begin{array}{cc}A_{11} & A_{12} / 2 \\ { }^{t} A_{12} / 2 & A_{22}\end{array}\right) \in \mathcal{H}_{n_{1}+n_{2}}\left(\mathbb{Z}_{p}\right) \cap \mathrm{GL}_{n_{1}+n_{2}}\left(\mathbb{Q}_{p}\right)$

with

$A_{11} \in \mathcal{H}_{n_{1}}\left(\mathbb{Z}_{p}\right), \quad A_{22} \in \mathcal{H}_{n_{2}}\left(\mathbb{Z}_{p}\right), \quad A_{12} \in M_{n_{1}, n_{2}}\left(\mathbb{Z}_{p}\right)$.

Let $m$ be the rank of $A$. Assume that $2 A_{11} \in \mathrm{GL}_{n_{1}}\left(\mathbb{Z}_{p}\right)$. Then we have

$$
\begin{aligned}
& F_{p}^{(m)}(A, X) \\
& \quad=F_{p}^{\left(m-n_{1}\right)}\left(A_{22}-\frac{1}{4} A_{11}^{-1}\left[A_{12}\right], \xi_{p}\left(A_{11}\right) p^{n_{1} / 2} X\right) .
\end{aligned}
$$

Corollary 3.4. Let $n_{1}$ and $n_{2}$ be positive even integers. Let

$$
A=\left(\begin{array}{cc}
A_{11} & A_{12} / 2 \\
{ }^{t} A_{12} / 2 & A_{22}
\end{array}\right) \in \mathcal{H}_{n_{1}+n_{2}}(\mathbb{Z})_{>0}
$$

with

$A_{11} \in \mathcal{H}_{n_{1}}(\mathbb{Z})_{>0}, \quad A_{22} \in \mathcal{H}_{n_{2}}(\mathbb{Z})_{>0}, \quad A_{12} \in M_{n_{1}, n_{2}}(\mathbb{Z})$.

Let $p_{0}$ be a prime number. Let $m$ be the rank of $A$. Assume that $2 A_{11} \in \mathrm{GL}_{n_{1}}\left(\mathbb{Z}_{p}\right)$ for any prime number $p \neq p_{0}$ and $2 A_{22} \in \mathrm{GL}_{n_{2}}\left(\mathbb{Z}_{p_{0}}\right)$. Then we have

$$
\begin{aligned}
\prod_{p} & F_{p}^{(m)}(A, X) \\
= & F_{p_{0}}^{\left(m-n_{2}\right)}\left(A_{11}-\frac{1}{4} A_{22}^{-1}\left[{ }^{t} A_{12}\right], \chi_{A_{22}}\left(p_{0}\right) p_{0}^{n_{2} / 2} X\right) \\
& \times \prod_{p \neq p_{0}} F_{p}^{\left(m-n_{1}\right)}\left(A_{22}-\frac{1}{4} A_{11}^{-1}\left[A_{12}\right], \chi_{A_{11}}(p) p^{n_{1} / 2} X\right) .
\end{aligned}
$$

Now to make the computation in Section 4 smooth, we give an explicit form of $F_{p}^{(1)}(A, X)$ and $F_{p}^{(2)}(A, X)$ in the case $\operatorname{deg} A=2$ (e.g., [Katsurada 05]).
Proposition 3.5. Let

$$
A=\left(\begin{array}{cc}
a_{11} & a_{12} / 2 \\
a_{12} / 2 & a_{22}
\end{array}\right) \in \mathcal{H}_{2}(\mathbb{Z})_{\geq 0}
$$

Put $e=e_{A}=\operatorname{gcd}\left(a_{11}, a_{12}, a_{22}\right)$.

(1) Assume rank $A=1$. Then we have

$$
F_{p}^{(1)}(A, X)=\sum_{i=0}^{\operatorname{ord}_{p}\left(e_{A}\right)}(p X)^{i}
$$

(2) Assume $A>0$. Then we have

$$
\begin{aligned}
F_{p}^{(2)}(A, X) & \sum_{i=0}^{\operatorname{ord}_{p}\left(e_{A}\right)}\left(p^{2} X\right)^{i} \sum_{j=0}^{\operatorname{ord}_{p}\left(\mathfrak{f}_{A}\right)-i}\left(p^{3} X^{2}\right)^{j}-\chi_{A}(p) p X \\
& \sum_{i=0}^{\operatorname{ord}_{p}\left(e_{A}\right)}\left(p^{2} X\right)^{i} \sum_{j=0}^{\operatorname{ord}_{p}\left(\mathfrak{f}_{A}\right)-i-1}\left(p^{3} X^{2}\right)^{j}
\end{aligned}
$$

Now we give an explicit form of a differential operator in the case of degree 2 due to Ibukiyama. Let $y_{1}, y_{2}, y_{3}$ be variables, and for a positive even integer $l$ put

$$
\begin{aligned}
& G_{l}\left(y_{1}, y_{2}, y_{3} ; t\right) \\
& \quad=\frac{1}{R\left(y_{1}, y_{2}, y_{3} ; t\right)^{(2 l-5) / 2}\left(\Delta_{0}\left(y_{1}, y_{2} ; t\right)^{2}-4 y_{3} t^{2}\right)^{1 / 2}}
\end{aligned}
$$

where

$$
\Delta_{0}\left(y_{1}, y_{2} ; t\right)=1-y_{1} t+y_{2} t^{2}
$$

and

$$
\begin{aligned}
& R\left(y_{1}, y_{2}, y_{3} ; t\right) \\
& \quad=\left(\Delta_{0}\left(y_{1}, y_{2} ; t\right)+\left(\Delta_{0}\left(y_{1}, y_{2} ; t\right)^{2}-4 y_{3} t^{2}\right)^{1 / 2}\right) / 2 .
\end{aligned}
$$

Write

$$
G_{l}\left(y_{1}, y_{2}, y_{3} ; t\right)=\sum_{m=0}^{\infty} G_{l, m}\left(y_{1}, y_{2}, y_{3}\right) t^{m}
$$

and define a polynomial map

$$
Q_{l, m}\left(\left(\begin{array}{cc}
W_{1} & W_{2} \\
{ }^{t} W_{2} & W_{4}
\end{array}\right)\right)
$$

from $S_{4}(\mathbb{C})$ to $\mathbb{C}$ by

$$
\begin{aligned}
& Q_{l, m}\left(\left(\begin{array}{cc}
W_{1} & W_{2} \\
{ }^{t} W_{2} & W_{4}
\end{array}\right)\right) \\
& \quad=G_{l, m}\left(\operatorname{det} W_{2}, \operatorname{det} W_{1} \operatorname{det} W_{4}, \operatorname{det}\left(\begin{array}{cc}
W_{1} & W_{2} \\
{ }^{t} W_{2} & W_{4}
\end{array}\right)\right),
\end{aligned}
$$


where $W_{1}, W_{4} \in S_{2}(\mathbb{C})$ and $W_{2} \in M_{2}(\mathbb{C})$. Furthermore, define a polynomial map $P_{l, m}\left(X_{1}, X_{2}\right)$ from $M_{2}(\mathbb{C}) \times$ $M_{2}(\mathbb{C})$ to $\mathbb{C}$ by

$$
P_{l, m}\left(X_{1}, X_{2}\right)=Q_{l, m}\left(\left(\begin{array}{cc}
X_{1}{ }^{t} X_{1} & X_{1}{ }^{t} X_{2} \\
X_{2}{ }^{t} X_{1} & X_{2}{ }^{t} X_{2}
\end{array}\right)\right) .
$$

Then by [Ibukiyama 99] we have the following proposition.

Proposition 3.6. $P_{l, m}\left(X_{1}, X_{2}\right)$ satisfies [Katsurada 08, Section 3, Conditions $D-1$ to $D-3]$.

Furthermore, by a direct but rather elaborate calculation we have the following result.

Proposition 3.7. We have

$$
\begin{aligned}
& G_{l, m}\left(y_{1}, y_{2}, y_{3}\right) \\
& =\sum_{n=0}^{[m / 2]}\left(\begin{array}{c}
2 n+l-5 / 2 \\
n
\end{array}\right) y_{3}^{n} \\
& \times \sum_{\nu=0}^{[(m-2 n) / 2]}\left(-y_{2}\right)^{\nu}\left(\begin{array}{c}
l+m-\nu-5 / 2 \\
m-2 n-\nu
\end{array}\right) \\
& \times\left(\begin{array}{c}
m-2 n-\nu \\
\nu
\end{array}\right)\left(2 y_{1}\right)^{m-2 n-2 \nu},
\end{aligned}
$$

where

$$
\left(\begin{array}{c}
s \\
m
\end{array}\right)=\frac{\prod_{i=1}^{m}(s-i+1)}{m !}
$$

We note that $G_{l, m}\left(y_{1}, y_{2}, y_{3}\right) \in 2^{-m} \mathbb{Z}\left[y_{1}, y_{2}, y_{3}\right]$. Let

$$
\begin{aligned}
& \mathcal{G}_{l, m}
\end{aligned}
$$

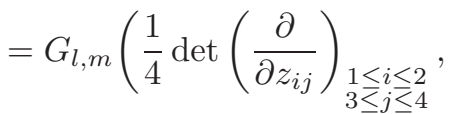

$$
\begin{aligned}
& \operatorname{det}\left(\frac{\tilde{\partial}}{\partial z_{i j}}\right)_{1 \leq i, j \leq 2} \operatorname{det}\left(\frac{\tilde{\partial}}{\partial z_{i j}}\right)_{3 \leq i, j \leq 4} \\
& \left.\operatorname{det}\left(\frac{\tilde{\partial}}{\partial z_{i j}}\right)_{1 \leq i, j \leq 4}\right) \text {, }
\end{aligned}
$$

and for $f \in C^{\infty}\left(\mathbb{H}_{4}\right)$ we define $\tilde{\mathcal{G}}_{l, m}(f)$ by

$$
\tilde{\mathcal{G}}_{l, m}(f)=\mathcal{G}_{l, m}(f)_{Z_{12}=0},
$$

where we write

$$
Z=\left(\begin{array}{cc}
Z_{1} & Z_{12} \\
{ }^{t} Z_{12} & Z_{2}
\end{array}\right) \text { with } Z_{1}, Z_{2} \in \mathbb{H}_{2}, Z_{12} \in M_{2}(\mathbb{C})
$$

By [Ibukiyama 99], $\tilde{\mathcal{G}}_{l, m}$ is a constant multiple of $\stackrel{\circ}{\mathcal{D}} \underset{2, l}{m}$. Namely, we have

$$
\tilde{\mathcal{G}}_{l, m}=d_{l, m} \stackrel{\circ}{\mathcal{D}} \underset{2, l}{m}
$$

with some $d_{l, m}$. To obtain an exact value of $d_{l, m}$, put $w=z_{13} z_{24}-z_{14} z_{23}$. Then for any integer $s$ we have

$$
\frac{1}{4} \operatorname{det}\left(\frac{\partial}{\partial z_{i j}}\right)_{1 \leq i \leq 2,3 \leq j \leq 4}\left(w^{s}\right)=C_{2}(s / 2) w^{s-1}
$$

and

$$
\operatorname{det}\left(\frac{\tilde{\partial}}{\partial z_{i j}}\right)_{1 \leq i, j \leq 4}\left(w^{s}\right)=C_{2}(s / 2) C_{2}((s-1) / 2) w^{s-2}
$$

and

$$
\operatorname{det}\left(\frac{\tilde{\partial}}{\partial z_{i j}}\right)_{1 \leq i, j \leq 2} \operatorname{det}\left(\frac{\tilde{\partial}}{\partial z_{i j}}\right)_{3 \leq i, j \leq 4}\left(w^{s}\right)=0,
$$

where $C_{2}(s)=s(s+1 / 2)$.

Thus for a positive even integer $m$ we have

$$
\begin{gathered}
\tilde{\mathcal{G}}_{l, m}\left(w^{m}\right) \\
=\prod_{\mu=1}^{m} C_{2}(\mu / 2) \sum_{n=0}^{m / 2}\left(\begin{array}{c}
2 n+l-5 / 2 \\
n
\end{array}\right) \\
\times\left(\begin{array}{c}
l+m-5 / 2 \\
m-2 n
\end{array}\right) 2^{m-2 n} \\
=\prod_{\mu=1}^{m} C_{2}(\mu / 2)\left(\begin{array}{c}
2 l+2 m-5 \\
m
\end{array}\right) .
\end{gathered}
$$

Here we have used the formula

$$
\sum_{n=0}^{m / 2}\left(\begin{array}{c}
s-m+2 n \\
n
\end{array}\right)\left(\begin{array}{c}
s \\
m-2 n
\end{array}\right) 2^{m-2 n}=\left(\begin{array}{c}
2 s \\
m
\end{array}\right)
$$

for $s \in \mathbb{C}$. On the other hand, we have

$$
\stackrel{\circ}{\mathcal{D}}_{2, l}^{m}\left(w^{m}\right)=\prod_{\mu=1}^{m} C_{2}(\mu / 2) C_{2}(l-2+m-\mu / 2)
$$

(cf. [Katsurada 08, Section 3]), and therefore we have

$$
d_{l, m}=\frac{\left(\begin{array}{c}
2 l+2 m-5 \\
m
\end{array}\right)}{\prod_{\mu=1}^{m} C_{2}(l-2+m-\mu / 2)} .
$$

Now for a positive even integer $l \leq k-2$ put

$$
\begin{aligned}
\tilde{\Lambda}(f, l, \underline{\mathrm{St}})= & \frac{\left(\begin{array}{c}
2 k-5 \\
k-l-2
\end{array}\right)}{2^{4 k-2 l-9}} \Gamma(l+1) \Gamma(k+l-2) \\
& \times \Gamma(k+l-1) \frac{L(f, l, \underline{\mathrm{St}})}{\langle f, f\rangle(2 \pi)^{2 k+3 l-3}} .
\end{aligned}
$$


Now let $l$ be an even integer such that $4 \leq l \leq k-2$, and put

$$
\begin{aligned}
& \tilde{\mathcal{E}}_{4, l, k}\left(z_{1}, z_{2}\right) \\
& =(-1)^{l / 2+1} 2^{-2}(2 \pi \sqrt{-1})^{2(l-k)}(l-2) \tilde{\mathcal{G}}_{l, k-l}\left(E_{4, l}\right)\left(z_{1}, z_{2}\right),
\end{aligned}
$$

where $z_{1}, z_{2} \in \mathbb{H}_{2}$. Then we note that $E_{4, l}(Z, 0)$ belongs to $\mathfrak{M}_{l}\left(\Gamma^{(4)}\right)$, and $\tilde{\mathcal{E}}_{4, l, k}\left(z_{1}, z_{2}\right)$ belongs to $\mathfrak{\Theta}_{k}\left(\Gamma^{(2)}\right) \otimes$ $\mathfrak{\Theta}_{k}\left(\Gamma^{(2)}\right)$.

We note that

$$
\tilde{\Lambda}(f, l-2, \underline{\mathrm{St}})=d_{l, k-l} \Lambda(f, l-2, \underline{\mathrm{St}})
$$

and

$$
\tilde{\mathcal{E}}_{4, l, k}\left(z_{1}, z_{2}\right)=d_{l, k-l} \mathcal{E}_{4, l, k}\left(z_{1}, z_{2}\right) .
$$

Now for positive definite half-integral matrices $A_{1}$ and $A_{2}$ of degree 2, let $\epsilon_{l, k}\left(A_{1}, A_{2}\right)$ be as in Section 2, and put $\tilde{\epsilon}_{l, k}\left(A_{1}, A_{2}\right)=d_{l, k} \epsilon_{l, k-l}\left(A_{1}, A_{2}\right)$. Furthermore, for each positive definite half-integral matrix $A_{1}$ put

$$
\tilde{\mathcal{F}}_{l, k ; A_{1}}\left(z_{2}\right)=\sum_{A_{2} \in \mathcal{H}_{2}(\mathbb{Z})_{>0}} \tilde{\epsilon}_{l, k}\left(A_{1}, A_{2}\right) \mathbf{e}\left(\operatorname{tr}\left(A_{2} z_{2}\right)\right) .
$$

Then we have $\tilde{\mathcal{F}}_{l, k ; A_{1}}\left(z_{2}\right)=d_{l, k-l} \mathcal{F}_{l, k ; A_{1}}\left(z_{2}\right)$, and therefore we have

$$
\tilde{\mathcal{E}}_{4, l, k}\left(z_{1}, z_{2}\right)=\sum_{A_{1} \in \mathcal{H}_{2}(\mathbb{Z})_{>0}} \tilde{\mathcal{F}}_{l, k ; A_{1}}\left(z_{2}\right) \mathbf{e}\left(\operatorname{tr}\left(A_{1} z_{1}\right)\right) .
$$

Let $p_{0}$ be a prime number. Assume that $2 A_{1} \in \mathrm{GL}_{2}\left(\mathbb{Z}_{p}\right)$ for any prime number $p \neq p_{0}$ and $2 A_{2} \in \mathrm{GL}_{2}\left(\mathbb{Z}_{p_{0}}\right)$. Then we have

$$
\begin{aligned}
& \tilde{\epsilon}_{l, k}\left(A_{1}, A_{2}\right) \\
& \quad=\sum_{R \in M_{2}(\mathbb{Z})} \tilde{c}_{4, l}\left(\left(\begin{array}{cc}
A_{1} & R / 2 \\
{ }^{t} R / 2 & A_{2}
\end{array}\right)\right) \\
& \times G_{l, k-l}\left(\frac{1}{4} \operatorname{det} R, \operatorname{det} A_{1} \operatorname{det} A_{2}, \operatorname{det}\left(\begin{array}{cc}
A_{1} & R / 2 \\
{ }^{t} R / 2 & A_{2}
\end{array}\right)\right),
\end{aligned}
$$

where

$$
\begin{aligned}
\tilde{c}_{4, l}(A) & \\
= & (-1)^{l / 2+1}(l-2) F_{p_{0}}^{(m-2)} \\
\times & \left(A_{1}-\frac{1}{4} A_{2}^{-1}\left[{ }^{t} R\right], \chi_{A_{2}}\left(p_{0}\right) p_{0}^{l-m}\right) \\
\times & \prod_{p \neq p_{0}} F_{p}^{(m-2)}\left(A_{2}-\frac{1}{4} A_{1}^{-1}[R], \chi_{A_{1}}(p) p^{l-m}\right) \\
& \times\left\{\begin{array}{c}
L\left(3-l, \chi_{A}^{(m-2)}\right) \\
\text { if } m=4, \\
\zeta(5-2 l) \\
\text { if } m=3,
\end{array}\right.
\end{aligned}
$$

for

$$
A=\left(\begin{array}{cc}
A_{1} & R / 2 \\
{ }^{t} R / 2 & A_{2}
\end{array}\right)
$$

with $\operatorname{rank}(A)=m$. We note that $\tilde{\epsilon}_{l, k}\left(A_{1}, A_{2}\right)$ is a rational number and any prime divisor of its denominator is not greater than $(2 l-1)$ !.

Fix an $A_{1} \in \mathcal{H}_{2}(\mathbb{Z})_{>0}$ and a prime number $p$. We define $\epsilon_{l, k}\left(i, A_{1}, A\right)$ as follows:

$$
\begin{aligned}
\epsilon_{l, k}\left(0, A_{1}, A\right)= & \tilde{\epsilon}_{l, k}\left(A_{1}, A\right), \\
\epsilon_{l, k}\left(i, A_{1}, A\right)= & \epsilon_{l, k}\left(i-1, A_{1}, p A\right) \\
& +p^{2 k-3} \epsilon_{l, k}\left(i-1, A_{1}, A / p\right) \\
& +p^{k-2} \sum_{D} \epsilon_{l, k}\left(i-1, A_{1}, A[D] / p\right),
\end{aligned}
$$

where

$$
U_{p}=\left(\begin{array}{ll}
1 & 0 \\
0 & p
\end{array}\right)
$$

and the sum is over $D \in \mathrm{GL}_{2}(\mathbb{Z}) U_{p} \mathrm{GL}_{2}(\mathbb{Z}) / \mathrm{GL}_{2}(\mathbb{Z})$. Let $\left\{f_{j}\right\}_{j=1}^{d}$ be an orthogonal basis of $\mathfrak{S}_{k}\left(\Gamma^{(2)}\right)$ consisting of Hecke eigenforms, and let $\lambda_{j}$ be the eigenvalue of $T(p)$ for $f_{j}$. Then by the Hecke theory of Siegel modular forms (e.g., [Andrianov 87]) we have

$$
\mathcal{F}_{l, k ; A_{1}} \mid T(p)^{i}\left(z_{2}\right)=\sum_{A_{2} \in \mathcal{H}_{2}(\mathbb{Z})_{>0}} \epsilon_{l, k}\left(i, A_{1}, A_{2}\right) \mathbf{e}\left(\operatorname{tr}\left(A_{2} z_{2}\right)\right)
$$

for any nonnegative integer $i$ and $A_{1} \in \mathcal{H}_{2}(\mathbb{Z})_{>0}$. Thus by Theorem 2.7 we have the following result.

Theorem 3.8. In addition to the above assumption, let $f=f_{1}, \lambda=\lambda_{1}, a\left(A_{1}\right)=a_{1}\left(A_{1}\right), a(A)=a_{1}(A)$, $K=\mathbb{Q}(f)$, and for a positive even integer $l \leq$ $k-4$ put $e_{i}=\epsilon_{l+2, k}\left(i, A_{1}, A\right)$. Furthermore, let $\Phi(X)=\Phi_{T(p)}(X)=\sum_{i=0}^{d} b_{d-i} X^{i}$ be the characteristic polynomial of $T(p)$ in $\mathfrak{\Im}_{k}\left(\Gamma^{(2)}\right)$. Put $\tilde{\Lambda}^{*}(f, l, \underline{\mathrm{St}})=$ $N_{K / \mathbb{Q}}(\tilde{\Lambda}(f, l, \underline{\mathrm{St}})) N\left(\mathfrak{I}_{f}\right)^{2}$. Assume that $\Phi^{\prime}(\lambda) \neq 0$ and $a\left(A_{1}\right) a(A) \neq 0$. Then we have

$$
\begin{aligned}
\tilde{\Lambda}^{*}(f, l, \underline{\mathrm{St}})= & N_{K / \mathbb{Q}}\left(\frac{\sum_{i=0}^{d-1} \sum_{j=i}^{d-1} e_{d-1-j} b_{j-i} \lambda^{i}}{\Phi^{\prime}(\lambda)}\right) \\
& \times \frac{N\left(\mathfrak{I}_{f}\right)^{2}}{N_{K / \mathbb{Q}}\left(a\left(A_{1}\right) a(A)\right)} .
\end{aligned}
$$

\section{NUMERICAL EXAMPLES AND COMMENTS}

We compute the special values of the standard zeta functions using Mathematica. Let $\phi_{10,1}(\tau, z)$ and $\phi_{12,1}(\tau, z)$ be the Jacobi cusp forms in $J_{10,1}^{\text {cusp }}$ and $J_{12,1}^{\text {cusp }}$ in [Eichler and Zagier 85, p. 40], respectively. Here $\tau \in \mathbb{H}_{1}$ 


\begin{tabular}{|l|l|}
\hline$l$ & \multicolumn{1}{|c|}{$\boldsymbol{N}_{K / \mathbb{Q}}\left(\tilde{\Lambda}\left(\hat{\boldsymbol{f}}_{\boldsymbol{i}}, \boldsymbol{l}, \underline{\mathbf{S t}}\right) \mathbf{N}\left(\mathfrak{I}_{\hat{f}_{i}}\right)^{2}\right.$} \\
\hline 2 & $3^{5} \cdot 5^{5} \cdot 7 \cdot 11 \cdot 13^{2} \cdot 17^{2} \cdot 23^{2} \cdot 29 \cdot 31 / 2^{46} \cdot D$ \\
4 & $3^{7} \cdot 5^{3} \cdot 7^{2} \cdot 11 \cdot 13 \cdot 17^{2} \cdot 23 \cdot 29 \cdot 31 \cdot 173 \cdot 443 / 2^{39} \cdot D$ \\
6 & $3^{6} \cdot 5^{3} \cdot 11^{2} \cdot 13^{3} \cdot 17^{2} \cdot 29 \cdot 31 \cdot 227 \cdot 1381069 / 2^{31} \cdot D$ \\
8 & $3^{9} \cdot 5^{5} \cdot 7 \cdot 11 \cdot 13^{2} \cdot 17^{2} \cdot 23 \cdot 29 \cdot 31 \cdot 21347 \cdot 58169 / 2^{22} \cdot D$ \\
10 & $3^{8} \cdot 5^{6} \cdot 7^{2} \cdot 13^{3} \cdot 17^{2} \cdot 19 \cdot 23 \cdot 31 \cdot 863 \cdot 3673 \cdot 3426433 / 2^{17} \cdot D$ \\
12 & $3^{6} \cdot 5^{3} \cdot 7^{2} \cdot 11 \cdot 17^{2} \cdot 23 \cdot 29 \cdot 37 \cdot 293 \cdot 691^{2} \cdot 33721 \cdot 96875477 / 2^{4} \cdot D$ \\
14 & $2^{7} \cdot 3^{8} \cdot 5^{4} \cdot 7^{3} \cdot 11 \cdot 13^{2} \cdot 17 \cdot 23^{2} \cdot 29^{2} \cdot 31 \cdot 467196139 \cdot 541368271 / D$ \\
16 & $2^{25} \cdot 3^{14} \cdot 5^{4} \cdot 7 \cdot 11 \cdot 13 \cdot 17 \cdot 23 \cdot 29^{2} \cdot 31^{2} \cdot 67 \cdot 1699 \cdot 3617^{2} \cdot 296551 / D$ \\
\hline
\end{tabular}

TABLE 1. Standard zeta values of the S-K lift of weight 20.

and $z \in \mathbb{C}$. Furthermore, let $E_{1, k}(\tau)$ be the Eisenstein series of weight $k$ with respect to $\Gamma^{(1)}$ defined in Section 2 , and put $E_{k}(\tau)=\zeta(1-k)^{-1} E_{1, k}(\tau)$. Then it is well known that $E_{4}^{a}(\tau) E_{6}(\tau)^{b} \phi_{j, 1}(\tau, z)$, for $a, b \geq 0$, $j=10,12,4 a+6 b+j=k$, form a basis of $J_{k, 1}^{\text {cusp }}$. Let

$$
A_{0}=\left(\begin{array}{cc}
1 & 1 / 2 \\
1 / 2 & 1
\end{array}\right), \quad A_{1}=\left(\begin{array}{cc}
1 & 0 \\
0 & 1
\end{array}\right)
$$

and

$$
A_{2}=\left(\begin{array}{cc}
2 & 1 / 2 \\
1 / 2 & 2
\end{array}\right) \text {. }
$$

Furthermore, we denote by $\mathfrak{\Xi}_{k}\left(\Gamma^{(2)}\right)^{*}$ the Maass subspace of $\mathfrak{\Im}_{k}\left(\Gamma^{(2)}\right)$.

We have $\operatorname{dim} \mathfrak{\Xi}_{20}\left(\Gamma^{(2)}\right)=3$ and $\operatorname{dim} \mathfrak{S}_{38}\left(\Gamma^{(1)}\right)=2$. Let $f_{1}, f_{2}$ be the basis of $\mathfrak{S}_{38}\left(\Gamma^{(1)}\right)$ consisting of primitive forms. For $i=1,2$ let $\lambda_{i}=48(-2025+\sqrt{D})$ and $48(-2025-\sqrt{D})$ with $D=63737521$. Then $\lambda_{i}$ are the eigenvalues of $T(2)$ with respect to $f_{i}$. They satisfy the equation $X^{2}+194400 X^{2}-137403408384=0$, and $\mathbb{Q}\left(f_{i}\right)=\mathbb{Q}\left(\lambda_{i}\right)=K$ with $K=\mathbb{Q}(\sqrt{D})$ (cf. [Hida and Maeda 97]). Put $\theta_{i}=\lambda_{i} / 96$. Then $\theta_{i}$ satisfies the equation $g(X):=X^{2}+2025 X-14909224=0$.

The discriminant of $g(X)$ is $D$. Thus the discriminant of $\mathbb{Q}(\sqrt{D})$ is $D$, and the ring of integers in $\mathbb{Q}(\sqrt{D})$ is $\mathbb{Z}\left(\theta_{1}\right)$. Let $h_{1}(\tau, z)=E_{4}(\tau) E_{6}(\tau) \phi_{10,1}(\tau, z)$ and $h_{2}(\tau, z)=E_{4}(\tau)^{2} \phi_{12,1}(\tau, z)$. These form a basis of $J_{20,1}^{\text {cusp }}$. Put $g_{i}=\mathcal{V} h_{i}$ for $i=1,2$. Then these form a basis of $\mathfrak{S}_{20}\left(\Gamma^{(2)}\right)^{*}$, whose $A_{0}$ th Fourier coefficient is 1 . Furthermore, for $i=1,2$ put $\hat{f}_{i}=-230 g_{1}+\left(-4862-\theta_{i}\right) g_{2}$.

Then $\hat{f}_{i}$ is the Saito-Kurokawa lift of $f_{i}$, whose $A_{0}$ th Fourier coefficient is $a_{\hat{f}_{i}}\left(A_{0}\right)=-5092-\theta_{i}$. We note that we have $\hat{f}_{i}=\chi_{20}^{(i)} / 2$ for $i=1,2$, where $\chi_{20}^{(1)}$ and $\chi_{20}^{(2)}$ are the eigenforms in [Kurokawa 78]. Then we have $a_{\hat{f}_{i}}\left(A_{1}\right)=-10\left(4816+\theta_{i}\right)$.

Furthermore, we have $\lambda_{\hat{f}_{i}}(T(2))=\lambda_{i}+3 \cdot 2^{18}$. Then

$$
N_{K / \mathbb{Q}}\left(a_{\hat{f}_{i}}\left(A_{0}\right)\right)=2^{2} \cdot 3^{4} \cdot 5 \cdot 19 \cdot 23
$$

and

$$
N_{K / \mathbb{Q}}\left(a_{\hat{f}_{i}}\left(A_{1}\right)\right)=-2^{5} \cdot 3 \cdot 5^{2} \cdot 23 \cdot 2659 .
$$

By a simple computation we have $N\left(\mathfrak{I}_{\hat{f}_{i}}\right)=2^{5} \cdot 3^{2} \cdot 5 \cdot 23$.

Let $\Upsilon 20$ be the cuspidal Hecke eigenform in [Skoruppa 92]. It is the unique (up to a constant) Hecke eigenform in $\mathfrak{\Xi}_{20}\left(\Gamma^{(2)}\right)$ that is not a Saito-Kurokawa lift. We note that $\Upsilon 20=\chi_{20}^{(3)} / 2$, where $\chi_{20}^{(3)}$ is the Hecke eigenform in [Kurokawa 78]. Then $\hat{f}_{1}, \hat{f}_{2}$, and $\Upsilon 20$ form an orthogonal basis of $\mathfrak{S}_{20}\left(\Gamma^{(2)}\right)$. We have $\mathfrak{I}_{\Upsilon 20}=1$, $a_{\Upsilon 20}\left(A_{0}\right)=1$, and $a_{\Upsilon 20}\left(A_{1}\right)=2^{2}$. Furthermore, we have $\lambda_{\Upsilon 20}(T(2))=-2^{8} \cdot 3^{2} \cdot 5 \cdot 73$. Thus by Theorem 3.8 , we have the information contained in Tables 1 and 2.

We have $\operatorname{dim} \mathfrak{S}_{22}\left(\Gamma^{(2)}\right)=4$ and $\operatorname{dim} \mathfrak{S}_{42}\left(\Gamma^{(1)}\right)=3$. Let $f_{1}, f_{2}, f_{3}$ be the basis of $\mathfrak{S}_{42}\left(\Gamma^{(1)}\right)$ consisting of primitive forms. For $i=1,2,3$ let $\lambda_{i}$ be the eigenvalues of the $T(2)$ with respect to $f_{i}$. Then they satisfy the equation

$$
\begin{gathered}
X^{3}+344688 X^{2}-6374982426624 X \\
-520435526440845312=0
\end{gathered}
$$

and $\mathbb{Q}\left(f_{i}\right)=\mathbb{Q}\left(\lambda_{i}\right)$ (cf. [Hida and Maeda 97]). Put $\theta_{i}=$ $\lambda_{i} / 48$ for $i=1,2,3$. Then $\theta_{i}$ is also an algebraic integer, and it satisfies the following equation:

$$
\begin{aligned}
g(X) & :=X^{3}+7181 X^{2}-2766919456 X-4705905729536 \\
& =0 .
\end{aligned}
$$

\begin{tabular}{|l|l|}
\hline $\boldsymbol{l}$ & \multicolumn{1}{|c|}{$\tilde{\boldsymbol{\Lambda}}(\mathbf{\Upsilon} \mathbf{2 0}, \boldsymbol{l}, \mathbf{S t})$} \\
\hline 2 & $3^{3} \cdot 5 \cdot 7 \cdot 11 \cdot 23 \cdot 29 \cdot 31 / 2^{28}$ \\
4 & $3^{2} \cdot 5^{2} \cdot 13 \cdot 23 \cdot 29 \cdot 31 \cdot 113 / 2^{25}$ \\
6 & $3^{4} \cdot 5 \cdot 7 \cdot 29 \cdot 31 \cdot 7549 / 2^{17}$ \\
8 & $3^{3} \cdot 5 \cdot 7^{2} \cdot 11 \cdot 29 \cdot 31 \cdot 37 \cdot 4861 / 2^{16}$ \\
10 & $3 \cdot 5 \cdot 7 \cdot 31 \cdot 283 \cdot 617 \cdot 4098371 / 2^{13}$ \\
12 & $3^{4} \cdot 5^{2} \cdot 7^{2} \cdot 11 \cdot 29 \cdot 31 \cdot 337 \cdot 91909 / 2^{6}$ \\
14 & $2^{4} \cdot 3^{4} \cdot 7^{2} \cdot 13 \cdot 29 \cdot 12893 \cdot 2166127$ \\
16 & $2^{11} \cdot 3^{6} \cdot 5^{3} \cdot 13 \cdot 23 \cdot 29 \cdot 347162819$ \\
\hline
\end{tabular}

TABLE 2. Standard zeta values of $\Upsilon 20$. 
The discriminant of $g(X)$ is $-2^{10} \cdot 3^{4} \cdot 5^{2} \cdot 7^{2} \cdot 1465869841$. 578879197969 .

Let

$$
\begin{aligned}
& h_{1}(\tau, z)=E_{4}(\tau)^{3} \phi_{10,1}(\tau, z), \\
& h_{2}(\tau, z)=E_{6}(\tau)^{2} \phi_{10,1}(\tau, z), \\
& h_{3}(\tau, z)=E_{4}(\tau) E_{6}(\tau) \phi_{12,1}(\tau, z) .
\end{aligned}
$$

Then these form a basis of $J_{22,1}^{\text {cusp }}$. Put $g_{i}=\mathcal{V} h_{i}$ for $i=$ $1,2,3$. Then these form a basis of $\mathfrak{\subseteq}_{22}\left(\Gamma^{(2)}\right)^{*}$ whose $A_{0}$ th Fourier coefficient is 1 . Furthermore, for $i=1,2,3$ put

$$
\begin{aligned}
\hat{f}_{i}= & 1155\left(435776+31 \theta_{i}\right) g_{1}-220\left(4760624+79 \theta_{i}\right) g_{2} \\
& +\left(286270336-60563 \theta_{i}+\theta_{i}^{2}\right) g_{3} .
\end{aligned}
$$

Then $\hat{f}_{i}$ is the Saito-Kurokawa lift of $f_{i}$ whose $A_{0}$ th Fourier coefficient $a_{\hat{f}_{i}}\left(A_{0}\right)$ is $-257745664-42138 \theta_{i}+\theta_{i}^{2}$. Then we have $a_{\hat{f}_{i}}\left(A_{1}\right)=10\left(395073536-64248 \theta_{i}+\theta_{i}^{2}\right)$ and $a_{\hat{f}_{i}}\left(A_{2}\right)=-352\left(-3767171584-182733 \theta_{i}+\theta_{i}^{2}\right)$. Let $\theta=\theta_{1}, \hat{f}=\hat{f}_{1}, \lambda=\lambda_{1}$, and $K=\mathbb{Q}\left(f_{1}\right)$. Put $R_{1}=$ $1155(435776+31 \theta), R_{2}=-220(4760624+79 \theta)$, and $R_{3}=$ $286270336-60563 \theta+\theta^{2}$. For elements $u_{1}, u_{2}, \ldots, u_{r}$ of $K$ we temporarily denote by $\left\langle u_{1}, u_{2}, \ldots, u_{r}\right\rangle$ the $\mathbb{Z}$-module generated by $u_{1}, u_{2}, \ldots, u_{r}$. Then

$$
\left[\left\langle 1, \theta, \theta^{2}\right\rangle:\left\langle R_{1}, R_{2}, R_{3}\right\rangle\right]=2^{6} \cdot 3^{3} \cdot 5^{3} \cdot 7^{2} \cdot 11^{3} \cdot 13 \cdot 157
$$

and

$$
\left[\left\langle R_{1}, R_{2}, R_{3}\right\rangle:\left\langle a_{\hat{f}}\left(A_{0}\right), a_{\hat{f}}\left(A_{1}\right), a_{\hat{f}}\left(A_{2}\right)\right\rangle\right]=2^{8} 3^{4} .
$$

By observing the Fourier coefficients of $h_{1}(\tau, z)$, $h_{2}(\tau, z)$, and $h_{3}(\tau, z)$, we see that

$$
\operatorname{det}\left(\begin{array}{ccc}
a_{g_{1}}(A) & a_{g_{1}}(B) & a_{g_{1}}(C) \\
a_{g_{2}}(A) & a_{g_{2}}(B) & a_{g_{2}}(C) \\
a_{g_{3}}(A) & a_{g_{3}}(B) & a_{g_{3}}(C)
\end{array}\right)
$$

is divided by $2^{8} 3^{4}$ for any $A, B, C \in \mathcal{H}_{2}(\mathbb{Z})_{>0}$. Thus $\left[\left\langle R_{1}, R_{2}, R_{3}\right\rangle: \mathfrak{I}_{\hat{f}}\right]$ is divided by $2^{8} 3^{4}$.

Thus we have $\left\langle a_{\hat{f}}\left(A_{0}\right), a_{\hat{f}}\left(A_{1}\right), a_{\hat{f}}\left(A_{2}\right)\right\rangle=\mathfrak{I}_{\hat{f}}$ and

$$
\left[\left\langle R_{1}, R_{2}, R_{3}\right\rangle: \mathfrak{I}_{\hat{f}}\right]=2^{8} \cdot 3^{4}
$$

Now using the "round 2 method," we can find an element $\eta=\left(5984+5805 \theta+\theta^{2}\right) / 10080$ in $\mathfrak{D}_{K}$ such that $1, \theta, \eta$ form an integral basis of a $p$-maximal order of $\mathfrak{D}_{K}$ for $p=2,3,5,7$ (cf. [Cohen 93]). We have

$$
\left[\langle 1, \theta, \eta\rangle:\left\langle 1, \theta, \theta^{2}\right\rangle\right]=2^{5} \cdot 3^{2} \cdot 5 \cdot 7,
$$

and therefore the discriminant $D_{K}$ of $K$ is not divisible by $2 \cdot 3 \cdot 5 \cdot 7$. Thus, we have $D_{K}=-D$ with
$D=1465869841 \cdot 578879197969$ and $\mathfrak{D}_{K}=\langle 1, \theta, \eta\rangle$. This has also been examined with MAgma by M. Kida. The author thanks him for his kind help. Thus we have

$$
N\left(\mathfrak{I}_{\hat{f}}\right)=2^{19} \cdot 3^{9} \cdot 5^{4} \cdot 7^{3} \cdot 11^{3} \cdot 13 \cdot 157 .
$$

Furthermore, we have

$$
\lambda_{\hat{f}}(T(2))=\lambda+3 \cdot 2^{20},
$$

$N_{K / \mathbb{Q}}\left(a_{\hat{f}}\left(A_{0}\right)\right)=-2^{14} \cdot 3^{13} \cdot 5^{4} \cdot 7^{4} \cdot 11^{3} \cdot 13 \cdot 157 \cdot 1213$

and

$N_{K / \mathbb{Q}}\left(a_{\hat{f}}\left(A_{1}\right)\right)=-2^{24} \cdot 3^{8} \cdot 5^{5} \cdot 7^{3} \cdot 11^{3} \cdot 13 \cdot 157 \cdot 1447 \cdot 2437$.

Let $\Upsilon 22$ be the cuspidal Hecke eigenform in [Skoruppa 92]. It is the unique (up to a constant) Hecke eigenform in $\mathfrak{\Xi}_{22}\left(\Gamma^{(2)}\right)$ that is not a Saito-Kurokawa lift. Then $\hat{f}_{1}, \hat{f}_{2}, \hat{f}_{3}$, and $\Upsilon 22$ form an orthogonal basis of $\mathfrak{\subseteq}_{22}\left(\Gamma^{(2)}\right)$ and $\mathbb{Q}\left(\hat{f}_{i}\right)=\mathbb{Q}\left(f_{i}\right)$. We note that

$$
N_{K_{i} / \mathbb{Q}}\left(\tilde{\Lambda}\left(\hat{f}_{i}, l, \underline{\mathrm{St}}\right)\right) N\left(\mathfrak{I}_{\hat{f}_{i}}\right)^{2}=N_{K / \mathbb{Q}}(\tilde{\Lambda}(\hat{f}, l, \underline{\mathrm{St}})) N\left(\mathfrak{I}_{\hat{f}}\right)^{2}
$$

for any $i$.

We have $\mathfrak{I} \Upsilon 22_{2}=1, a_{\Upsilon 22}\left(A_{0}\right)=1$, and $a_{\Upsilon 22}\left(A_{1}\right)=$ $-2^{2} \cdot 3$. Furthermore, we have $\lambda_{\Upsilon 22}(T(2))=-2^{8} \cdot 3 \cdot 5$. 577 . Thus by Theorem 3.8, we have the information in Tables 3 and 4 .

Finally, we offer some comments. First, observing Tables 1 and 4, we note that prime factors of the $l$ th Bernoulli number and the norm of the algebraic part of $L\left(f_{i}, l+k-2\right) L\left(f_{i}, l+k-1\right)$ appear in the numerator of $N_{K / \mathbb{Q}}\left(\tilde{\Lambda}\left(\hat{f}_{i}, l, \underline{\mathrm{St}}\right) N\left(\mathfrak{I}_{\hat{f}_{i}}\right)^{2}\right.$. For example, the prime factor 43867 of $N_{K / \mathbb{Q}}\left(\tilde{\Lambda}\left(\hat{f}_{i}, 18, \underline{\mathrm{St}}\right) N\left(\mathfrak{I}_{\hat{f}_{i}}\right)^{2}\right.$ in Table 3 is a prime factor of the numerator of the 18th Bernoulli number, and the prime factors 13553776667 and 365257 of it appear in the norm of the algebraic parts of $L\left(f_{i}, 38\right)$ and $L\left(f_{i}, 39\right)$, respectively (cf. [Stein 04]). This is not so surprising, because we have

$$
L\left(\hat{f}_{i}, l, \underline{\mathrm{St}}\right)=\zeta(l) L\left(f_{i}, l+k-2\right) L\left(f_{i}, l+k-1\right)
$$

for $f_{i} \in \mathfrak{\Xi}_{2 k-2}\left(\Gamma^{(1)}\right)$.

Next we comment on our conjecture in [Katsurada 08]. Let $f$ be a Hecke eigenform in $\mathfrak{\Xi}_{k}\left(\Gamma^{(n)}\right)$ and let $M$ be a subspace of $\mathfrak{S}_{k}\left(\Gamma^{(n)}\right)$ stable under the Hecke operators $T \in \mathbf{L}_{n}^{\prime}$. Assume that $M$ is contained in $(\mathbb{C} f)^{\perp}$, where $(\mathbb{C} f)^{\perp}$ is the orthogonal complement of $\mathbb{C} f$ in $\mathfrak{S}_{k}\left(\Gamma^{(n)}\right)$ with respect to the Petersson product. A prime ideal $\mathfrak{P}$ of $\mathfrak{D}_{\mathbb{Q}(f)}$ is called a congruence prime of $f$ with respect to $M$ if there exists a Hecke eigenform $g \in M$ such that

$$
\lambda_{f}(T) \equiv \lambda_{g}(T) \bmod \tilde{\mathfrak{P}}
$$




\begin{tabular}{|c|c|}
\hline$l$ & $N_{K_{i} / \mathbb{Q}}\left(\tilde{\Lambda}\left(\hat{f}_{i}, l, \underline{\mathrm{St}}\right)\right) N\left(\mathfrak{I}_{\hat{f}_{i}}\right)^{2}$ \\
\hline 2 & $3^{9} \cdot 5^{4} \cdot 7 \cdot 11^{3} \cdot 13^{5} \cdot 17^{2} \cdot 19^{3} \cdot 23^{2} \cdot 29^{2} \cdot 31^{2} \cdot 37 / 2^{78} \cdot D$ \\
\hline 4 & $\begin{array}{l}3^{12} \cdot 5^{2} \cdot 7^{4} \cdot 11^{2} \cdot 13^{3} \cdot 17^{2} \cdot 19^{2} \cdot 29^{2} \cdot 31^{2} \cdot 37 \cdot 151 \cdot 1601 \cdot 6551 \\
\times 7951 / 2^{69} \cdot 1423 \cdot D\end{array}$ \\
\hline 6 & $\begin{array}{l}3^{12} \cdot 5^{9} \cdot 11^{3} \cdot 13^{3} \cdot 17^{2} \cdot 19^{2} \cdot 23 \cdot 29^{2} \cdot 31^{2} \cdot 37 \cdot 137 \cdot 809 \\
\times 38029874887 / 2^{57} \cdot 7 \cdot 1423 \cdot D\end{array}$ \\
\hline 8 & $\begin{array}{l}3^{9} \cdot 5 \cdot 7^{5} \cdot 11 \cdot 13^{4} \cdot 17^{2} \cdot 19^{2} \cdot 23^{2} \cdot 29 \cdot 31^{2} \cdot 37 \cdot 84521 \cdot 8947751 \\
\times 699588169271 / 2^{41} \cdot 1423 \cdot D\end{array}$ \\
\hline 10 & $\begin{array}{l}3^{10} \cdot 5^{9} \cdot 7^{3} \cdot 11^{4} \cdot 13^{4} \cdot 17^{2} \cdot 19^{3} \cdot 23 \cdot 29 \cdot 31 \cdot 37^{2} \\
\times 1423469629 \cdot 27864526583393 / 2^{28} \cdot 1423 \cdot D\end{array}$ \\
\hline 12 & $\begin{array}{l}3^{12} \cdot 5 \cdot 11^{2} \cdot 13 \cdot 17 \cdot 19^{2} \cdot 23^{2} \cdot 29^{2} \cdot 31 \cdot 37 \cdot 691^{3} \cdot 953 \\
\times 243911 \cdot 4251563 \cdot 6617174324030971171 / 2^{10} \cdot 7 \cdot 1423 \cdot D\end{array}$ \\
\hline 14 & $\begin{array}{l}2^{6} \cdot 3^{12} \cdot 5^{5} \cdot 7^{8} \cdot 11^{3} \cdot 13^{4} \cdot 17^{2} \cdot 19^{2} \cdot 23 \cdot 29^{2} \cdot 31^{2} \cdot 37 \\
\times 150197 \cdot 318467 \cdot 1465187 \cdot 13894099 \cdot 63630191 / 1423 \cdot D\end{array}$ \\
\hline 16 & $2^{26} \cdot 3^{19} \cdot 5^{5} \cdot 7^{2} \cdot 11^{3} \cdot 13^{3} \cdot 19 \cdot 23 \cdot 29^{2} \cdot 31^{2} \cdot 37 \cdot 3617^{3}$ \\
\hline & $\times 1465869841 \cdot 2775014078857939 \cdot 22683897890722493 / 1423 \cdot D$ \\
\hline 18 & $\begin{array}{l}2^{59} \cdot 3^{25} \cdot 5^{15} \cdot 11 \cdot 13^{2} \cdot 17^{2} \cdot 19^{2} \cdot 23 \cdot 29^{2} \cdot 31^{2} \cdot 37^{2} \\
\times 107 \cdot 43867^{3} \cdot 365257 \cdot 13553776667 / 1423 \cdot D\end{array}$ \\
\hline
\end{tabular}

TABLE 3. Standard zeta values of the S-K lift of weight 22.

\begin{tabular}{|l|l|}
\hline $\boldsymbol{l}$ & \multicolumn{1}{|c|}{$\tilde{\boldsymbol{\Lambda}}(\mathbf{\Upsilon} \mathbf{2 2} ; \boldsymbol{l}, \underline{\mathbf{S t}})$} \\
\hline 2 & $3^{3} \cdot 5 \cdot 11 \cdot 23 \cdot 29 \cdot 31 \cdot 37 / 2^{32}$ \\
4 & $3^{4} \cdot 5 \cdot 11 \cdot 13 \cdot 29 \cdot 31 \cdot 37 \cdot 103 \cdot 157 / 2^{27} \cdot 1423$ \\
6 & $3^{6} \cdot 11 \cdot 29 \cdot 31 \cdot 37^{2} \cdot 485363 / 2^{24} \cdot 1423$ \\
8 & $3^{2} \cdot 29 \cdot 31 \cdot 37 \cdot 149 \cdot 3361493719 / 2^{18} \cdot 1423$ \\
10 & $3^{3} \cdot 5 \cdot 11 \cdot 37 \cdot 89 \cdot 1039 \cdot 2741 \cdot 3616027 / 2^{15} \cdot 1423$ \\
12 & $3^{4} \cdot 11^{2} \cdot 31 \cdot 37 \cdot 421 \cdot 254725279909 / 2^{8} \cdot 1423$ \\
14 & $3^{3} \cdot 7^{2} \cdot 11 \cdot 13 \cdot 31 \cdot 37 \cdot 733 \cdot 2131 \cdot 82625047 / 2 \cdot 1423$ \\
16 & $2^{5} \cdot 3^{7} \cdot 5 \cdot 11 \cdot 13 \cdot 19 \cdot 31 \cdot 37 \cdot 30293340159041 / 1423$ \\
18 & $2^{16} \cdot 3^{8} \cdot 5^{2} \cdot 7 \cdot 13 \cdot 17 \cdot 31 \cdot 37 \cdot 101 \cdot 439 \cdot 1049 \cdot 49991 / 1423$ \\
\hline
\end{tabular}

TABLE 4. Standard zeta values of $\Upsilon 22$.

for any $T \in \mathbf{L}_{n}^{\prime}$, where $\tilde{\mathfrak{P}}$ is some prime ideal of $\mathfrak{D}_{\mathbb{Q}(f) \mathbb{Q}(g)}$ lying above $\mathfrak{P}$. If $M=(\mathbb{C} f)^{\perp}$, we call $\mathfrak{P}$ simply a congruence prime of $f$

Now to explain our conjecture. For a normalized Hecke eigenform $f$ in $\mathfrak{S}_{2 k-2}\left(\Gamma^{(1)}\right)$ and a Dirichlet character $\chi$, let $L(f, s, \chi)$ be the Hecke $L$-function of $f$ twisted by $\chi$ defined by as follows:

$$
L(f, s, \chi)=\sum_{m=1}^{\infty} a_{f}(m) \chi(m) m^{-s} .
$$

In particular, if $\chi$ is the principal character, we write $L(f, s, \chi)$ as $L(f, s)$. Put $\Omega_{f}^{(+)}=(2 \pi \sqrt{-1})^{-1} L(f, 1)$ and $\Omega_{f}^{(-)}=(2 \pi \sqrt{-1})^{-2} L(f, 2)$. For $j= \pm, 1 \leq l \leq 2 k-3$ and a Dirichlet character $\chi$ such that $\chi(-1)=j(-1)^{l-1}$, put

$$
\mathbf{L}(f, l, \chi)=\frac{(2 \pi \sqrt{-1})^{-l} \Gamma(l) L(f, l, \chi)}{\Omega^{(j)}} .
$$

In particular, put $\mathbf{L}(f, l)=\mathbf{L}(f, l, \chi)$ if $\chi$ is the principal character. Then, in [Katsurada 08], we proposed the following conjecture:

Conjecture 4.1. Let $\mathfrak{\Re}$ be a prime ideal of $\mathbb{Q}(f)$ not dividing $(2 k-1)$ !. Then $\mathfrak{P}$ is a congruence prime of $\hat{f}$ with respect to $\left(\mathfrak{S}_{k}\left(\Gamma^{(2)}\right)^{*}\right)^{\perp}$ if and only if $\mathfrak{P}$ divides the $n u$ merator of $\mathbf{L}(f, k)$. 
We note that the "if" part of the above conjecture is a special case of Harder's conjecture [Harder 03].

Now look at Table 3 . in this case, the prime number 1423 appears in the denominator of

$$
N_{K_{i} / \mathbb{Q}}\left(\tilde{\Lambda}\left(\hat{f}_{i}, l, \underline{\mathrm{St}}\right)\right) N\left(\mathfrak{I}_{\hat{f}_{i}}\right)^{2}
$$

for $l=4, \ldots, 18$. We have

$$
1423=\mathfrak{P}_{i} \mathfrak{P}_{i}^{\prime}
$$

in $\mathfrak{D}_{\mathbb{Q}\left(f_{i}\right)}$, where

$$
\mathfrak{P}_{i}=\left\langle\lambda_{i}+967,1423\right\rangle
$$

and

$$
\mathfrak{P}_{i}^{\prime}=\left\langle\lambda_{i}^{2}+778 \lambda_{i}+660,1423\right\rangle .
$$

We have $\operatorname{deg} \mathfrak{P}_{i}=1$ and $\operatorname{deg} \mathfrak{P}_{i}^{\prime}=2$. Thus by [Katsurada 08 , Theorem 5.2], $\mathfrak{P}_{i}$ is a congruence prime of $\hat{f}_{i}$, and by a more careful analysis, we see that it is a congruence prime of $\hat{f}_{i}$ with respect to $\mathbb{C} \Upsilon 22$. In fact, we have

$$
\lambda_{\hat{f}_{i}}(T(2)) \equiv \lambda_{\Upsilon 22}(T(2)) \bmod \mathfrak{P}_{i} .
$$

Conversely, by a direct calculation, we see that there is no other congruence prime greater than 43 of $\hat{f}_{i}$ with respect to $\mathbb{C} \Upsilon 22$. On the other hand, according to the numerical table in [Stein 04], we have

$$
\begin{aligned}
& \left|N_{\mathbb{Q}(f) / \mathbb{Q}}(\mathbf{L}(f, 22))\right| \\
& \quad=\frac{11^{3} \cdot 17 \cdot 1423}{2^{23} \cdot 3^{18} \cdot 5^{10} \cdot 13 \cdot 29 \cdot 31 \cdot 37^{2} \cdot 137 \cdot 7481} .
\end{aligned}
$$

This implies that the conjecture in [Katsurada 08, Section 6$]$ is true in this case.

We note that the "if" part has been proved in [Brown 07] and [Katsurada 08] independently under certain conditions.

\section{ACKNOWLEDGMENTS}

The author would like to thank the referee for many valuable comments.

\section{REFERENCES}

[Andrianov 87] A. N. Andrianov. Quadratic Forms and Hecke Operators. New York: Springer, 1987.

[Böcherer 85] S. Böcherer. "Über die Fourier-Jacobi-Entwicklung Siegelscher Eisensteinreihen II." Math. Z. 189 (1985), 81-110.
[Böcherer and Schmidt 00] S. Böcherer and C. G. Schmidt. " $p$-adic Measures Attached to Siegel Modular Forms." Ann. Inst. Fourier 50 (2000), 1375-1443.

[Brown 07] J. Brown. "Saito-Kurokawa Lifts and Applications to the Bloch-Kato Conjecture." Compositio Math. 143 (2007), 290-322.

[Cohen 93] H. Cohen. A Course in Computational Algebraic Number Theory. New York: Springer, 1993.

[Eichler and Zagier 85] M. Eichler and D. Zagier. The Theory of Jacobi Forms. Boston: Birkhäuser, 1985.

[Garrett 84] P. Garrett. "Pullbacks of Eisenstein Series." In Automorphic Forms of Several Variables, Progress in Math. 46, pp. 114-137. Boston: Birkhäuser, 1984.

[Goto 98] K. Goto. "A Twisted Adjoint $L$-Value of an Elliptic Modular Form." J. Number Theory 73 (1998), 34-46.

[Harder 03] G. Harder. "A Congruence between a Siegel Modular Form and an Elliptic Modular Form." Preprint, 2003.

[Hida and Maeda 97] H. Hida and Y. Maeda. "Nonabelian Base Change for Totally Real Fields." Pacific J. Math., Olga Taussky-Todd Memorial Issue (1997), 189-218.

[Ibukiyama 99] T. Ibukiyama. "On Differential Operators on Automorphic Forms and Invariant Pluri-harmonic Polynomials." Comm. Math. Univ. St. Pauli 48 (1999), 103-118.

[Katsurada 99] H. Katsurada. "An Explicit Formula for Siegel Series." Amer. J. Math. 121 (1999), 415-452.

[Katsurada 05] H. Katsurada. "Special Values of the Standard Zeta Functions for Elliptic Modular Forms." Experiment. Math. 14 (2005), 27-45.

[Katsurada 08] H. Katsurada. "Congruence of Siegel Modular Forms and Special Values of Their Standard Zeta Functions." Math. Z. 259 (2008), 97-111.

[Kitaoka 84] Y. Kitaoka. "Dirichlet Series in the Theory of Quadratic Forms." Nagoya Math. J. 92 (1984), 73-84.

[Kitaoka 93] Y. Kitaoka. Arithmetic of Quadratic Forms, Cambridge Tracts Math. 106. Cambridge, UK: Cambridge Univ. Press, 1993.

[Kurokawa 78] N. Kurokawa. "Examples of Eigenvalues of Hecke Operators on Siegel Cusp Forms of Degree Two." Invent. Math. 49 (1978), 149-165.

[Mizumoto 91] S. Mizumoto. "Poles and Residues of Standard L-Functions Attached to Siegel Modular Forms." Math. Ann. 289 (1991) 589-612.

[Shimura 83] G. Shimura. "On Eisenstein Series." Duke Math. J. 50 (1983), 417-476.

[Skoruppa 92] N.-P. Skoruppa. "Computations of Siegel Modular Forms of Genus Two." Math. Comp. 58 (1992), 381398.

[Stein 04] W. A. Stein. The Modular Forms Data Base." Available online (http://modular.fas.harvard.edu/index. html), 2004. 
Hidenori Katsurada, Muroran Institute of Technology, 27-1 Mizumoto Muroran, 050-8585, Japan (hidenori@mmm.muroran-it.ac.jp)

Received May 14, 2008; accepted in revised form February 17, 2009. 